\title{
Avances de la investigación en educación y TIC en aulas hospitalarias
}

\section{Advances of Research in Education and ICT in Hospital Classrooms}

\author{
José Peirats Chacón ${ }^{1}$ \\ jose.peirats@uv.es \\ Jesús Granados Saiz \\ jegra@alumni.uv.es \\ Diana Morote Blanco \\ diamo2@alumni.uv.es \\ Universitat de València, España
}

\begin{abstract}
:
Lately the use of digital educational applications as a tool for the education of convalescent students has proliferated. However, few studies have comprehensively reviewed the use of information and communication technology (ICT) and other resources used in hospital teaching. This study performs an analysis of the progress made in existing research on this type of educational experiences, evaluating the impact of ICT and checking that hospitalized students may connect with the educational community and its environment. The methodology employed was a literature review which allowed us to organize, classify and analyze documentary sources on the subject of study. The results revealed the importance of the quality of connections with the outside world in projects of ICT integration and the development and impact of innovative educational applications. We conclude by highlighting that ICT has improved communication and coordination among schools, families and hospitals. It has also offered new ways
\end{abstract}

1 Dirección para correspondencia (correspondence address):

José Peirats Chacón. Dpto. de Didáctica y Organización Escolar. Universitat de València. Blasco Ibáñez, 30. 46010 Valencia (España). 
las TIC han mejorado la comunicación y coordinación curricular entre centros educativos, familia y hospital, además de ofrecer nuevas formas para aprender mediadas con materiales digitalizados.

Palabras clave:

Aulas hospitalarias; atención educativa hospitalaria; TIC; recurso didáctico electrónico. to learn which are mediated through digitized resources.

\section{Key words:}

Hospital teaching; ITC; educational care hospital; electronic teaching aid.

\section{Résumé:}

Dernièrement, l'utilisation des applications didactiques numériques s'est multipliée dans I'environnement éducatif des élèves convalescents. Cependant, les recherches qui étudient de maniéré globale les technologies de l'information et la communication (TIC) ainsi que les matériaux dans les salles d'hôpital, sont peu nombreuses. Pour cette raison, nous avons réalisé une analyse des progrès réalisés dans la recherche et le développement, sur des expériences éducatives. Pour cela, nous avons évalué l'impact des TIC et vérifié si les élèves hospitalisés pouvaient se connecter à la communauté éducative et leur environnement. La méthodologie réalisée s'est déroulée de la manière suivante. Tout d'abord, une révision bibliographique qui nous a permis d'organiser, de classifier et d'analyser les sources documentaires sur le sujet en question. II faut souligner, parmi les résultats, l'importance de la qualité des connexions avec le monde extérieur dans les projets d'intégration des TIC, le développement et l'impact des nouvelles applications didactiques. Pour terminer, nous avons remarqué que les TIC ont amélioré la communication et la coordination du programme d'études entre les centres éducatifs, les familles et l'hôpital. Le tout, en offrant des nouvelles manières d'apprendre à l'aide d'instruments et ressources numériques.

\section{Mots clés:}

Salles d'hôpital; attention éducative hospitalière; TIC; moyen d'enseignement électronique.

Fecha de recepción: 16-5-2017

Fecha de aceptación: 22-9-2017

\section{Introducción}

Las aulas hospitalarias constituyen un factor esencial en el desarrollo educativo, formativo y afectivo de una serie de niños y niñas que, debido a una enfermedad, pasan un tiempo prolongado de su vida en un hospital, alejados de su colegio y de su ambiente familiar y social. En las funciones que desarrollan no se encuentra sólo el profesorado, sino que un gran número de familias y personal sanitario dan lo mejor de cada uno de ellos para que este alumnado sienta mínimamente la ausencia de su ambiente natural y de sus compañeros, compañeras y profesorado (Peirats y Granados, 2015). 
Según Almeida (2010), se utiliza el término de aula hospitalaria cuando el aula es llevada al hospital con el objeto de permitir que los niños y niñas internados en una institución médica puedan continuar con sus estudios y cuenten con un espacio de recreación. Con esta medida las administraciones pretenden garantizar que el alumnado hospitalizado pueda continuar el proceso educativo y mejorar sus condiciones de estancia en el centro hospitalario (Prendes y Serrano, 2014).

Actualmente, las $\mathrm{AH}$ suelen ser espacios altamente valorados, aunque a veces también desconocidos, en las instalaciones de algunos hospitales. Así lo demuestra Silva (2012) en su estudio, al señalar que "para los niños y niñas, los padres y madres de familia, el personal de salud y los docentes, el aula tiene el poder de humanizar la experiencia de hospitalización y brindar beneficios a todos los implicados" (p. 152).

En consecuencia, esa atención específica y dirigida que proveen estas aulas resulta de vital importancia, ya que ofrecen la posibilidad de mantener ambientes enriquecidos de aprendizaje que permiten al sujeto acceder a oportunidades de aprendizaje formal e informal en contextos lúdicos y coherentes con su edad y momento evolutivo. Bajo esta línea de pensamiento, hablar de las TIC y de los materiales didácticos digitalizados como parte integral de ese ambiente, y en este caso especialmente con alumnado hospitalizado, no presupone plantear nada extraño o sorprendente.

Tal y como apuntan Adell y Castañeda (2010), las TIC son una ocasión para reflexionar y repensar el modelo educativo que tenemos en la actualidad, puesto que la escuela puede que no esté ofreciendo ni reflejando lo que la sociedad demanda. En otro estudio Rodríguez, Gromaz y Hoppe (2008) afirman que la tecnología facilita al estudiante la flexibilidad para acceder a los materiales de aprendizaje, la tutoría online o el apoyo de otros y otras estudiantes en cualquier momento, independientemente de los horarios del hospital o de las preferencias del mismo.

La revisión de la literatura que presentamos se ha organizado alrededor de las TIC en el entorno educativo hospitalario, concretamente en tres secciones: los beneficios académicos de las TIC y sus materiales en las $\mathrm{AH}$, el impacto de las mismas para la reincorporación a la clase de referencia y, por último, las conexiones que establecen con el mundo exterior. Nuestro propósito ha sido el de analizar y comprender el impacto que supone el uso de las tecnologías y los recursos digitalizados en estas aulas, como prolongación necesaria de la educación inclusiva, 
y comprobar que el alumnado hospitalizado no se convierte en un "islote" aislado de la comunidad educativa y su entorno familiar y social.

\section{Método}

Este texto toma la forma conocida como "estado del arte", definida por Vélez y Galeano (2002) como una modalidad de investigación documental que permite el estudio del conocimiento acumulado escrito dentro de un área específica; su finalidad es dar cuenta del sentido del material documental sometido a análisis, con el fin de revisar de manera detallada y cuidadosa los documentos que tratan sobre un tema específico.

Este estudio abordado nos ha permitido comprender los diferentes sistemas, herramientas y aplicaciones que comparten las aulas hospitalarias a nivel internacional y nacional en relación con las TIC. En el análisis efectuado, los artículos relacionados con las secciones de nuestro trabajo han sido seleccionados e identificados teniendo en cuenta distintos criterios de exclusión: los relacionados con el ámbito sanitario, e inclusión: artículos publicados en bases de datos y revistas científicas mundiales en el período 2007-2015, otras publicaciones estrechamente relacionadas, redactadas en inglés y español, y alumnado de cualquier edad, patología y sexo.

En la Figura 1 se representa esquemáticamente el proceso seguido, donde observamos por un lado SCOPUS y WOS con las características empleadas en su búsqueda y, por otro lado, DIALNET, REDALYC y G. SCHOLAR con sus respectivos campos de búsqueda. Tras aplicar estos factores, hemos obtenido un total de 40 registros que representa un índice de saturación correcto para realizar una revisión bibliográfica en base a los objetivos planteados.

Los textos han sido clasificados en base a los tres grandes constructos que nos guían en este trabajo de revisión bibliográfica sobre la aplicación de las TIC en AH: el beneficio académico que reportan al alumnado convaleciente, la calidad de las conexiones con el mundo exterior y su socialización y el gran reto de proporcionar una reincorporación a la vida escolar totalmente normalizada e inclusiva tras el período de hospitalización. Respecto a la información manejada, se ha utilizado los resultados de las investigaciones seleccionadas relacionados con cada una de las tres categorías propuestas. 


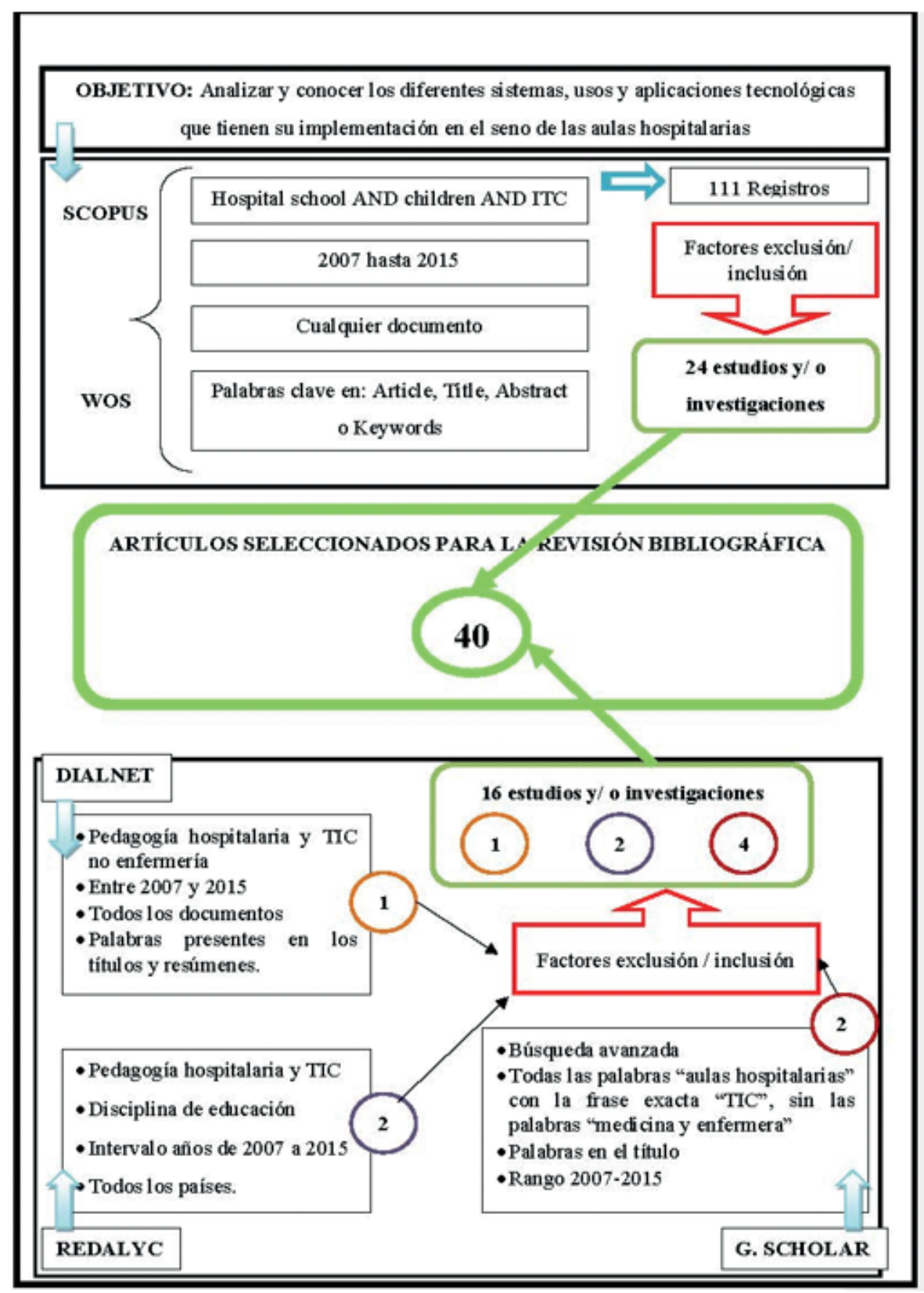

Figura 1. Proceso seguido de búsqueda y selección de la literatura empleada. 


\section{Resultados y discusión de los estudios}

Teniendo en cuenta la creciente importancia que suponen las TIC y la digitalización de los contenidos y los materiales didácticos curriculares en la educación de esta sociedad globalizada (Peirats, Gallardo, San Martín y Cortés, 2015; Rodríguez Rodríguez y Rodríguez Regueira, 2016), a raíz de la revisión de artículos e investigaciones seleccionados presentamos a continuación los resultados alcanzados relacionados con los ámbitos pedagógicos, didácticos y funcionales que facilitan la vida del niño y niña convaleciente.

\subsection{Beneficios académicos de las TIC en las aulas hospitalarias}

Dentro de la pedagogía hospitalaria es fundamental que las AH empleen las TIC como una herramienta de aprendizaje para reducir el letargo académico y mejorar el estado anímico del alumnado. Como exponen Serrano y Prendes (2011) las TIC no son solo un juego para cubrir un tiempo lúdico, todo lo contrario, pueden convertirse en un material pedagógico y didáctico si se emplean adecuadamente.

En este primer aspecto, destacan la coherencia de varios estudios (Chin y Tsuei, 2014; Hopkins, Nisselle, Zazryn y Green, 2013; Jones, McDougall y Robertson, 2009; Niselle, Hanns, Green y Jones, 2012); donde se manifiesta la problemática relacionada con los períodos prolongados de hospitalización y con la repetición de admisiones durante etapas del aprendizaje y del desarrollo, incluso en los puntos de transición psicoevolutiva. Este hecho coloca a los niños y niñas en un riesgo de bienestar social comprometido, fracaso escolar o un desenganche con la escuela, provocando resultados bajos a largo plazo, o la propia calidad de vida. Concretamente, Hopkins et al. (2013) establecen una serie de problemas entre los y las adolescentes en las fases de transición y en las decisiones importantes; destacan la pérdida de confianza en sí mismos, los estados anímicos alterados y un enlentecimiento del desarrollo emocional. Por ello han elaborado una escala de factores de riesgo y de protección para gestionar la capacidad de recuperación del compromiso educativo de los y las adolescentes. Incidiendo en los factores de protección, el estudio de Niselle et al. (2012) establece como esenciales: la proporción de netbooks en el hospital, la presencia de profesorado a tiempo completo, la interacción social entre el alumnado hospitalizado y un programa de 
enseñanza adaptado a cada individuo. Esta última característica es una de las principales aportaciones de Hopkins et al. (2013), al concluir que un aprendizaje basado en modelos personalizados ayudará a identificar las situaciones de riesgo educativo, poder aplicar estrategias de apoyo y evitar el retroceso académico.

En segundo lugar, una vez expuesta la importancia de las TIC para favorecer el progreso académico y de los factores de protección, trataremos aquellos resultados de las investigaciones para examinar los principales beneficios. Siguiendo con el estudio de Niselle et al. (2012) observamos que un $40,6 \%$ de los participantes otorgan a las TIC la categoría de ser el principal medio para mantenerse conectado con el día a día del trabajo escolar. Si en el tramo de la adolescencia el uso del correo electrónico y la Intranet supera el $70 \%$ de los encuestados, entendemos que el aspecto más importante para mantenerse al día con el trabajo escolar es probablemente el equipo tecnológico y los materiales didácticos digitalizados, siendo la única manera de conseguir la información necesaria para continuar con el proceso de enseñanza y aprendizaje.

En relación con los usos académicos, en muchas ocasiones se producen por intereses personales y mediante aprendizajes informales, así se puede comprobar en Niselle, Hanns, Green y Jones (2011) donde un sujeto consiguió aprender a tocar la guitarra mediante tutoriales de YouTube y en una web de partituras. Aunque este estudio revela también la sensación, por parte del alumnado, a no atribuir estas experiencias como beneficios educativos, sino más bien como pasatiempo o entretenimiento.

La mayoría de las interacciones con los netbooks se focalizan en búsquedas a través de Google y Wikipedia, otro punto de acción son las redes sociales. Encontramos los resultados de acceso a las redes telemáticas en Niselle et al. (2011), por parte del alumnado son: "Facebook $(48,6 \%)$, búsqueda de Google (34,3\%), YouTube $(20,0 \%)$ y Miniclip $(17,1 \%)$. También, los programas más utilizados fueron Microsoft Word (40,0\%), otros programas de Microsoft Office $(20,0 \%)$ y reproductores de audio MediaPlayer o iTunes (17.1\%)" (p. 13). En total el alumnado pasa una media de 3 horas diarias conectado a la red. No obstante, los datos finales indican que los y las convalecientes emplean los ordenadores tanto para actividades formales como informales de aprendizaje. En relación con el 25\% de los participantes del estudio de Niselle et al. (2011), que manifiestan su desacuerdo con las actividades informales de 
aprendizaje a través de la TIC, hemos revisado tres estudios que acercan el proceso de enseñanza y aprendizaje mediante software, aplicaciones y juegos digitales al alumnado convaleciente.

Según Pivec (2007), la estrategia del aprendizaje basado en juegos digitales aumenta la motivación del alumnado y ofrece oportunidades para la interacción y la comunicación entre los mismos. En la misma línea, Chin y Tsuei (2014) han elaborado dos juegos digitales multimodales (DGBL) que han conseguido proporcionar oportunidades de aprendizaje, mantener la motivación, desarrollar habilidades académicas según el ritmo del alumnado, contactar con sus iguales del hospital y mejorar la cooperación entre ellos. Los datos de este estudio muestran, finalmente, una aceptación positiva de las familias, ya que lo valoran como un excelente sistema de aprendizaje. El éxito del DGBL se debe al modelo de integración de experiencias y actividades, al sistema de recompensas y a las normas de ambos juegos que elevan la motivación por aprender del alumnado (Chin y Tsuei, 2014).

Es evidente que el factor motivacional a través de las TIC incide positivamente en el progreso académico, y así también se muestra en el estudio realizado por Hopkins et al. (2013), en el que utilizando el marco de factores de protección desarrollan dos experiencias con TIC, una de ellas a través de un Proyecto BLOG y otra mediante el juego de Angry Birds, para aprender el Teorema de Pitágoras. Estos dos casos demuestran la fortaleza que tienen las TIC y sus materiales didácticos en entornos hospitalarios y, sin lugar a duda, el desarrollo de las tareas en línea y del hardware están facilitando dicha tarea.

En otro estudio (Wilkie y Jones, 2008) donde los participantes lamentaron la pérdida de explicaciones por parte del profesorado, se integraron las TIC permitiendo al alumnado ausente participar de forma sincrónica en las actividades de aprendizaje realizadas por el equipo docente del centro de origen. Se pudo llevar a cabo a través de pizarras digitales interactivas (PDI) y el uso de la videoconferencia y, en el mismo, se registraron por parte del profesorado una cierta reticencia a este compromiso educativo, no siendo así hacia el acceso a la explicación de forma sincrónica o asincrónica, al permitir una grabación para un visionado posterior.

Con objeto de minimizar los efectos adversos de la hospitalización, Serradas Fonseca (2011) ha diseñado un software en la línea de los anteriores, aunque el aprendizaje se centra en el ámbito procedimental del 
conocimiento, donde el alumnado pone en funcionamiento las acciones ejecutivas. Considerando la propuesta y sus resultados se promueve la participación y sobre todo resuelve las necesidades del alumnado hospitalizado durante largos períodos de tiempo. Además, Aprendo en el Hospital es considerado por los agentes educativos como un software rápido y atractivo para la vida de un hospital.

La mayoría de los estudios de investigación, señalan que el mantenimiento de la vida normalizada puede disminuir la ansiedad y aumentar la sensación de control personal. En ese sentido, Niselle et al. (2011) señalan que "el suministro de netbooks contribuye a un ambiente normalizado y a una conexión con el mundo 'exterior' que proporciona la oportunidad de comprometerse con el aprendizaje de este alumnado" (p. 17). Sin embargo, hemos encontrado una interesante excepción a esta generalización en el estudio presentado por Jones, McDougall y Robertson (2009) en el que, partiendo de la premisa de que la mera presencia de tecnología en el aula no cambia nada, solicitaron a personal docente que modificasen su metodología de trabajo en el aula. Y, en concordancia con la problemática descrita, se indica en el trabajo que el principal problema o factor de riesgo en sus estudios estuvo relacionado con los niveles de conocimiento y experiencia que el profesorado tiene sobre el uso de las TIC.

En conclusión, los estudios demuestran que las infraestructuras, sistemas y aplicaciones son un hecho evidente y factible, pero sigue siendo un reto involucrar de una manera eficaz al profesorado en el proceso (Wilkie y Jones, 2008). Según estos últimos autores, hay evidencias de que muchos profesores y profesoras no utilizan las TIC como parte integral de su práctica diaria en el aula, lo que dificulta muchísimo estas experiencias educativas inclusivas en coordinación con las AH. De la revisión de investigaciones anteriores se deduce que existe una cierta tendencia a considerar los materiales y sistemas tecnológicos, obviando las metodologías de uso de las TIC. Consecuentemente, una de las futuras preocupaciones debe ser integración contextualizada de las TIC diseñando software y aplicaciones tras decidir los criterios pedagógicos y metodológicos.

\subsection{Impacto de las TIC en la reincorporación a la clase de referencia}

El alumnado con enfermedades crónicas experimenta largos períodos de ausencia de la escuela, por lo que puede retroceder en sus estudios. La 
literatura analizada sugiere que los programas que tratan de mantener al hospitalizado en contacto con sus estudios y compañeros y compañeras tienen como objetivo primordial una reincorporación ajustada a las emociones tanto de la clase como del alumnado (Ellis et al., 2013; Hopkins, et al., 2013; Lambert, Coad, Hicks y Glacken, 2014). Varios estudios subrayan que los niños con enfermedades crónicas se encuentran con un mayor índice de riesgo para la adaptación social y una menor aceptación por parte de sus iguales en el regreso a la escuela después de largas ausencias (Dunlap, Kagan, Arnold y Gottschlich, 2013; Leger y Campbell, 2008; Wilkie y Jones, 2008).

Los estudios de Dunlap et al. (2013) y Ellis et al. (2013) expresan en sus conclusiones la profunda preocupación del enfermo en relación con las posibles reacciones de sus iguales en cuanto a la apariencia física o las secuelas del tratamiento. Por tanto, ambos sostienen que los programas que promueven el contacto y buscan apoyo entre pares durante las largas ausencias mejoran las relaciones, promueven el desarrollo social y reducen el absentismo, una vez que el estudiante regresa a la escuela. No obstante, en el segundo estudio, se recoge en sus resultados que un $50 \%$ de las familias señalaron que la clase se acostumbró a la apariencia física del paciente y mejoraron el retorno de su hijo, encontrando una confianza y autoestima superior a los inicios de su hospitalización.

Según los resultados de Hopkins et al. (2013), el primer objetivo en este apartado es construir un marco de referencia que se constituya como una estructura de apoyo, incluyendo a la familia, escuela, hospital y la comunidad. En los dos casos expuestos en el estudio, se consiguió la comunicación con sus iguales, un aumento de la motivación y un compromiso educativo lo que repercutió positivamente en las fases de transición escuela-hospital.

Analizando los resultados del programa Remember me (Dunlap et al, 2013), observamos que se obtuvo un $100 \%$ de respuestas positivas por parte de los compañeros y compañeras, a través de diversos recursos: correos electrónicos, mensajes de texto, vídeos de presentación y diferentes formatos de comunicación que lograron aumentar los niveles del sentimiento de pertenencia del ausente. Este programa integra las potentes redes sociales, con el objetivo de maximizar la comunicación, que fueron muy destacadas por los encuestados. Sin embargo, el elemento más alabado y aceptado por todos fue un peluche en forma de osito, colocado en la silla del convaleciente. Los investigadores apunta- 
ron, positivamente, que en una gran cantidad de vídeos y mensajes se le mencionaba en términos muy emocionantes $y$, por su parte, los familiares indicaron que era un símil muy acertado, llegando a calificarlo como "un trofeo de vida" para su hijo enfermo.

En los trabajos de Dunlap et al. (2013) y Ellis et al. (2013), encontramos otros factores importantes como son la participación de la institución escolar y el beneficio reportado al grupo-clase donde se escolariza el hospitalizado. En la mayoría de los casos analizados, se benefician de un ambiente escolar saludable y optimista frente a una atmosfera de temor, piedad y/o actitudes discriminatorias. Concretamente, en Ellis et al. (2013), el programa aplicado ayuda a establecer una estrecha relación con la escuela y genera un gran beneficio en la reincorporación a la escuela, así como el éxito social y académico. Las familias informaron que la videoconferencia les proporcionó un sentido de normalidad y de conexión con el mundo exterior, impulsando el estado de ánimo del convaleciente y ofreciéndole una perspectiva esperanzadora. Otra de las ventajas que incluye la red de videoconferencias del estudio son las relaciones estrechas con el profesorado; en cambio, en evaluaciones académicas posteriores se comprobó que no tuvo efectos notables sobre la progresión académica del paciente. El profesorado fue unánime al informar que la clase disfrutó y el alumnado se sentía entusiasmado con el Connectivity Project, desarrollando empatía y comprensión. Sin embargo, al igual que en Remember me, una parte del profesorado y familias describen el impacto educativo como "limitado" debido a las dificultades técnicas, problemas de programación o adversidades médicas.

A lo dicho sumamos un estudio centrado en el aprendizaje académico del alumnado a través de PDI y videoconferencias constantes para seguir la dinámica del centro de referencia (Wilkie y Jones, 2008). Entre sus resultados, encontramos la necesidad de abrir una ventana en el proceso de enseñanza-aprendizaje a través de comunicaciones sincrónicas y asincrónicas. Los investigadores, en su discusión, argumentan la necesidad de utilizar las tecnologías para continuar el progreso educativo y no aumentar la brecha curricular, ya que advierten del momento de la incorporación como el instante significativo para la normalización e inclusión del alumnado. En otro estudio, donde se promueve la creación de salas tecnológicas con un doble fin, el ocio y la conexión con el mundo exterior, los autores (Lambert et al., 2014) sostienen que el convaleciente desea un número mayor de conexiones con el mundo 
exterior, destacando que este alumnado manifiesta la tristeza que les supone estar alejados de su familia, amigos, mascotas, profesores... En una de las encuestas, el $90 \%$ del alumnado apunta el uso de la tecnología para ampliar el registro de su socialización. La oportunidad para el intercambio de información y establecer vínculos con otros niños y niñas de la comunidad hospitalaria se ha demostrado que conlleva beneficios tangibles terapéuticos. Los resultados de este mismo estudio revelan que la disponibilidad y accesibilidad de las diversas actividades y espacios para activar el ocio interactivo, el entretenimiento, el juego social y el aprendizaje fueron de gran valor para la mejora en el retorno al colegio.

Finalmente señalar que el impacto de los programas de apoyo asume el potencial de implementarse en la comunidad escolar y ayudar en la consecución de una hoja de ruta para alcanzar la recuperación exitosa. En ese recorrido, serán significativos los primeros días donde el enfermo arrastra miedos, temores y creencias que se pueden abordar mediante conexiones diarias, comunicaciones interactivas, presencia e implicación del profesorado del centro de origen, seguimiento del trabajo escolar y un trabajo social con los compañeros y compañeras del aula. Los resultados globales de los estudios revisados indican que el uso de las TIC ayuda al convaleciente crónico a mantenerse en contacto con sus escuelas, alumnado y profesorado y facilitan su reincorporación.

\subsection{Conexiones con el mundo exterior a través de las TIC}

Para muchos estudiantes con malas condiciones de salud crónicas, el absentismo escolar es un efecto secundario inevitable del tratamiento médico, ya sea en el hospital o durante la recuperación en casa y son muchos los equipos multidisciplinares que se han propuesto el reducir el riesgo de desconexión durante estos períodos de ausencia para evitar el abandono escolar prematuro y su bajo rendimiento escolar (Hopkins, Wadley, Vetere, Fong y Green, 2014).

En primer lugar, es necesario destacar los trabajos que emplean la videoconferencia ya que suponen el inicio de la conexión entre el hospital y cualquier otro punto de interés del niño. En este sentido Ellis et al. (2013), además de Heidari, Kazemzadeh y Wadley (2015), han realizado investigaciones en torno a las videoconferencias, recibiendo valoraciones muy positivas por la capacidad de mantener relaciones sociales afectivas con altos niveles de entusiasmo y comprensión por parte del 
centro de referencia. No obstante, muchos participantes expresan que su ideal comunicativo serían las aplicaciones de videochat, para facilitar la sensación de presencia. El estudio de Heidari, et al. (2015) se centra en la valoración familiar del impacto que generan las TIC en familias con dificultades geográficas. En sus resultados señalan una cierta inestabilidad emocional; por un lado, porque las familias describen sentimientos como el temor o el miedo al recibir información acerca de la salud de su hijo o hija y también de frustración por verlos en una situación desfavorable. Sin embargo, las TIC les permiten no desatender al resto de la familia y seguir conectado al convaleciente.

Es evidente que la videoconferencia es una herramienta implementada en muchos hospitales y que ofrece unos resultados excelentes, creando un sentimiento de pertenencia y fomentando relaciones sociales a distancia, como en los sujetos del estudio anterior. No obstante, los avances tecnológicos han desarrollado nuevas y modernas opciones de conectar varios puntos geográficos. Este es el caso de la aplicación Presence, desarrollada por Hopkins et al. (2014), diseñada para permitir a pacientes, familias y compañeros y compañeras a desarrollar la sensación de "estar juntos", es decir, de presencia. La comunicación e interacción con esta aplicación es tan continua que ha producido resultados negativos en el profesorado y en el personal sanitario, debido al uso excesivo entre el alumnado del aula de referencia y el hospitalizado. En cambio, el convaleciente desarrolló el sentimiento de presencia y se mejoró el proceso de transición casa-escuela ya que, en muchos casos, se redujo el impacto físico de ver por primera vez el enfermo en clase. Con relación a este último aspecto, los registros de llamadas fallidas en el estudio de Wilkie y Jones (2010) son considerables debido al temor por mostrar la apariencia física a través de las TIC; aunque en otro estudio Nicholas, Fellner, Koller, Fontana y Brister (2011) mejoraron positivamente estos temores y sensaciones gracias a la comunicación digital.

Respecto al uso de dispositivos, encontramos el de los laptops como tecnología por excelencia (Nisselle et al. 2011) y en otro estudio con familiares se señala que la telefonía móvil ha alcanzado una mayor conectividad y conciencia de cercanía por la facilidad de su uso (Heidari et al. 2015). En ambos estudios, se afirma que la simple presencia de dispositivos móviles crea un ambiente normalizado y de seguridad tanto en los familiares como en los propios pacientes. En el trabajo de Hopkins et al. (2014) se emplean las tabletas para desarrollar la aplicación 
Presence, basada en la comunicación y las conexiones asincrónicas; el dispositivo fue ampliamente acogido por todos los participantes excepto por el profesorado integrado en centros con déficit tecnológico y altamente desmotivado.

Otro de los estudios muestra la capacidad de mantener los lazos escolares a través de un programa, Link'n Learn, con uso de videoconferencias, correo electrónico, mensajería instantánea y chats en línea. Entre sus resultados, Wilkie y Jones (2010) apuntan que el profesorado se mostraba contrario por cuestiones de economía temporal, aunque los investigadores señalan una cierta falta de implicación. Pero lo más destacable de este estudio, sin duda, es el elevado número de estudiantes que en sus entrevistas manifestaban haber perdido el hilo educativo por la desconfianza o la escasa voluntad del profesorado para conectarse con él.

Por otro lado, y debido a las nuevas exigencias de la sociedad, hemos encontrado tres trabajos que han implementado aspectos de la llamada tecnología ambiental. Según Antón, Mana, Muñoz y Koshutanski (2011) la tecnología ambiental (AMI) es un paradigma en el que la tecnología de la información se concreta en la construcción de redes de servicios y dispositivos que están conectados de forma dinámica y colaboran para ayudar a las personas en diferentes actividades. Del mismo modo, Vetere et al. (2012) menciona a las AMI como tecnologías periféricas que no exigen atención, que operan en el fondo de otra actividad primaria y que son muy eficaces para el intercambio de información tendiendo a promocionar el sentimiento de cercanía y presencia.

En el trabajo de Wadley, Vetere, Hopkins, Green y Kulik (2014) se integra la tecnología ambiental para compartir fotos del alumnado hospitalizado utilizando la aplicación Presence como software. La aceptación por parte de los convalecientes fue bien recibida, al igual que su profesorado, destacando en sus descripciones la dimensión fática de estas herramientas. Incluso para el profesorado, activo en el mantenimiento de la conexión del niño a la familia y al aprendizaje a través de tecnologías como el correo electrónico y mensajes de texto. La presencia de la tecnología ambiente fue contemplada como un complemento útil, ya que la mayoría afirmaba que podían mejorar la conexión entre sus estudiantes y el hospitalizado.

En otro trabajo, donde implementaban el programa LIFE, se evaluaba su beneficio en base a tres factores: el sueño, el estrés y el rendimiento escolar. El programa permite a los usuarios utilizar un monitor periférico 
como una ventana al mundo real dando una sensación aumentada del contacto remoto. Esta sensación se consigue por medio de la detección del usuario, de sus acciones y movimientos para mostrar su imagen remota. Antón et al. (2011) destacan en sus resultados la inmersión de los niños del hospital y la reducción del aislamiento social y del estrés que genera encontrarse hospitalizado. Es importante reseñar la capacidad del vídeo en streaming con el objetivo de dar un aliciente y mantener las rutinas escolares, aunque obviamente y como exponen en sus conclusiones, es limitado por ser una comunicación unidireccional.

Por último, en otra investigación sobre la aplicación de tecnología ambiental con cuatro estudios de caso, se ha utilizado un "orbe" brillante: un artefacto que conecta la red móvil del convaleciente con el aula de origen. No hay mensajes ni señales, simplemente se controla el color del dispositivo iluminándolo en mayor o menor medida. En los resultados Vetere et al. (2012) apuntan que las familias demandan una mayor comunicación directa y real para sus hijos e hijas, el profesorado se encuentra más cómodo al ser una tecnología menos invasiva para su día a día y sus iguales aumentaron la cantidad de intervenciones para hablar sobre el alumnado enfermo, lo que se tradujo en multitud de reflexiones y correos. Tras estos resultados estos autores señalan que la combinación de varias formas de conexión con el niño enfermo, desde las más tradicionales hasta las más actuales, tienen en común la capacidad de trascender los detalles físicos de la enfermedad para crear una sensación de estar presente en el aula y un sentimiento de unión a su grupo social.

El conocimiento obtenido en la revisión efectuada nos permite, entre otras cosas, vislumbrar algunas de las posibilidades más certeras para seguir mejorando las conexiones con el alumnado de las $\mathrm{AH}$. En consecuencia, dejamos constancia de una constante en todas las investigaciones, y es que el éxito en las situaciones en las que el profesorado planificó el uso de la herramienta fue mucho mayor que en las que no se realizó ningún tipo de previsión.

\section{Conclusiones}

A partir de los resultados de la revisión mostramos ahora aquellos hallazgos más interesantes en cada uno de los aspectos analizados. En líneas generales señalamos que las TIC y sus materiales didácticos presentan 
las siguientes ventajas: suponen un impacto positivo en la emoción y la conducta del niño, mejora la gestión del aprendizaje, almacenan contenidos, personalizan y adaptan actividades a sus necesidades, permiten la interacción sincrónica o asincrónica y acercan el currículo al hospitalizado. A las que Blanco (2015) añade otras ventajas relacionadas con el uso de las TIC, como son el acceso directo e ilimitado, la actualización personal, la multiplicidad de tareas, el trabajo autónomo y el beneficio en la resiliencia.

En referencia a los beneficios académicos, las TIC reportan aspectos favorables para la educación del convaleciente, siendo de gran incidencia para el proceso de enseñanza-aprendizaje. Concretamente, el simple hecho de incluir soportes y aplicaciones en las $\mathrm{AH}$ es considerado como un hecho esencial, evitando de ese modo el posible retroceso académico. En este punto mencionaremos que la existencia de las TIC promueve tanto un uso formal como lúdico de las mismas. Las TIC han evolucionado y de ahí que hayan surgido nuevas experiencias educativas que, a priori, puedan catalogarse de informales debido a su interfaz, pero, desde nuestra revisión, somos conscientes de la presencia del componente académico que, por ejemplo, poseen los juegos multimodales de Chin y Tsuei (2014) o el juego de Angry Birds en Hopkins, et al. (2013). Se hace evidente que las creaciones de nuevos sistemas suponen de inmediato nuevos usos, creando un clima académico en las AH. En consecuencia, dotando de progreso académico al alumnado hospitalizado será mucho más fácil desbloquear la incorporación al centro educativo de origen.

En la gran mayoría de las referencias analizadas, hemos encontrado como elemento negativo la apariencia física de los convalecientes en el regreso al centro. Es el motivo por el que se ha valorado de forma positiva la posibilidad de crear comunicaciones audiovisuales mediante videoconferencias, que favorecen las comunicaciones sincrónicas con alumnado y profesorado, disminuyendo así el sentimiento de soledad y aumentando la empatía de los iguales. Por tanto, mantener el contacto diario con sus iguales y con la familia supone un ítem de protección, lo que propiciará un espectro de relaciones sociales interactivas, además de mantener la relación académica y el proceso de enseñanza a través de las TIC, facilitando en gran medida una vuelta normalizada al centro de origen.

Siguiendo con la última sección, nos fijamos en las conexiones del mundo exterior a través de las TIC. En ese aspecto, el conjunto de pro- 
gramas de videoconferencia revisados ha mostrado un aumento en los niveles de optimismo y entusiasmo en cada conexión que sus sujetos realizaron. No obstante, en varios trabajos relacionados hemos encontrado, como aspecto negativo, que un uso excesivo de los soportes tecnológicos invadía en muchas ocasiones la vida escolar del aula de referencia o el trabajo profesional del personal sanitario. Debemos de ser cautelosos en este sentido, ya que es posible correr el riesgo de violar la privacidad del paciente o de otros agentes implicados. Sin embargo, sostenemos que el nivel de satisfacción del profesorado implicado, en gran parte de las propuestas tecnológicas, ha sido elevado.

No obstante, han aparecido nuevos caminos tecnológicos capaces de solventar dicho problema, hablamos de la tecnología ambiental. Este tipo de tecnología puede complementar a las TIC, en ningún caso sustituirla. En definitiva, todos los soportes son capaces de favorecer la comunicación sincrónica o asincrónica, pero lo que realmente es importante y ha sido demostrado es la capacidad de planificar y coordinar eficazmente las conexiones con el mundo exterior desde el hospital.

De todo lo dicho establecemos unas metaconclusiones, como resultado final de nuestro trabajo, en relación a las TIC en las AH. Como son que median del proceso de enseñanza y aprendizaje, personalizan y adaptan actividades a sus necesidades, permiten una interacción sincrónica o asincrónica, aproximan al niño hospitalizado el currículum, reducen el sentimiento de aislamiento y ansiedad, aumentan los niveles de seguridad en las familias y catalizan las relaciones sociales y las emociones positivas. Los planes de formación relacionados con la integración de las TIC en las AH se destacan como una de las grandes barreras para su implementación, ya que en la bibliografía revisada no se ha encontrado información al respecto. Por este motivo, el uso pedagógico y la formación específica es una de las tareas pendientes es este peculiar contexto educativo.

En cuanto a las limitaciones encontradas, es destacable la dificultad de encontrar artículos que se centren en el estudio del currículo adaptado mediante $\mathrm{TIC}$ en aulas hospitalarias. Consideramos relevante abrir una línea de investigación en este sentido, ya que las TIC nos ayudan en infinidad de aspectos, pero sería propedéutico establecer estudios de casos en relación con los ajustes curriculares mediados con TIC siendo como es, en muchas ocasiones, el único medio de enlace entre el aula de origen y la hospitalaria. 
Para acabar consideramos que las TIC están cambiando de forma radical la vida cotidiana entre los distintos contextos educativos, al permitir mejorar la comunicación y coordinación entre centros educativos, familias y hospital, además de disminuir el aislamiento del menor al que ahora, afortunadamente, se le ofrecen nuevas formas de aprender $y$ relacionarse.

\section{Referencias bibliográficas}

Adell, J. y Castañeda, L. (2010). Los Entornos Personales de Aprendizaje (PLEs): una nueva manera de entender el aprendizaje. Recuperado de https://digitum.um.es/jspui/ handle/10201/17247

Almeida, P. (2010). Análisis de las metodologías aplicadas en el proceso de enseñanzaaprendizaje en las aulas hospitalarias en niños de 4-6 años. Quito: Universidad Politécnica Salesiana.

Antón, P., Mana, A., Muñoz, A. y Koshutanski, H. (2011). Live interactive frame technology alleviating children stress and isolation during hospitalization. In Ambient Assisted Living. Springer Berlin Heidelberg. DOI: 10.1007/978-3-642-21303-8_13.

Blanco, J. D. P. G. (2015). Uso de las Tecnologías de la información y la comunicación (TIC) en Aulas hospitalarias: Tendencias y Aplicaciones. Trabajo presentado en el Primer Congreso Internacional de Pedagogía Hospitalaria, Colombia.

Chin, J. C. y Tsuei, M. (2009). Multi-user Narrative Sharing System for Children with Chronic Illness. In Advanced Learning Technologies, 2009. ICALT 2009. Ninth IEEE International Conference on. DOI: 10.1109/ICALT.2009.154

Chin, J. C. y Tsuei, M. (2014). A Multi-modal Digital Game-based Learning Environment for Hospitalized Children with Chronic Illnesses. Journal of Educational Technology \& Society, 17(4), 366-378. Recuperado de http://www.jstor.org/stable/jeductechsoci.17.4.366

Dunlap, D., Kagan, R. J., Arnold, S. y Gottschlich, M. (2013). "Remember Me" Program: Bridging the Gap Between Hospital and School. Journal of Burn Care \& Research, 34(2), e99-e103. DOI: 10.1097/BCR.0b013e3182853e97

Ellis, S. J., Drew, D., Wakefield, C. E., Saikal, S. L., Punch, D. y Cohn, R. J. (2013). Results of a Nurse-Led Intervention Connecting Pediatric Cancer Patients From the Hospital to the School Using Videoconferencing Technologies. Journal of Pediatric Oncology Nursing, 30(6), 333-341. DOI: 10.1177/1043454213514633

González González, C. S., Toledo, P., Padrón, M., Santos, E. y Cairos, M. (2013). TANGO: H: Creating Active Educational Games for Hospitalized Children. Management Intelligent Systems. Springer International Publishing. DOI 10.1007/978-3-31900569-0_17

Guirao-Goris, J. A., Olmedo Salas, A. y Ferrer Ferrandis, E. (2008). El artículo de revisión. Revista Iberoamericana de Enfermería Comunitaria, 1(1), 1-25.

Heidari, A., Kazemzadeh, Y. y Wadley, G. (2015). ICT's Effect on Parents' Feelings of Pre- 
sence, Awareness, and Connectedness during a Child's Hospitalization. Australasian Conference on Information Systems, Adelaide (Australia).

Hopkins, L., Nisselle, A., Zazryn, T. y Green, J. (2013). Hospitalised adolescents: A framework for assessing educational risk. Youth Studies Australia, 32(1), 37.

Hopkins, L., Moss, J., Green, J. y Strong, G. (2014). Embedding learning in a paediatric hospital: changing practice and keeping connected. International Journal of Inclusive Education, 18(3), 312-321.

Hopkins, L., Wadley, G., Vetere, F., Fong, M. y Green, J. (2014). Utilising technology to connect the hospital and the classroom: Maintaining connections using tablet computers and a 'Presence' App. Australian Journal of Education, 0(0), 1-19. DOI: 10.1177/0004944114542660.

Jones, A., McDougall, A. y Robertson, M. (2009). Strategies for enabling teachers to utilise ICT for addressing educational disadvantage resulting from prolonged school absence. En PL Jeffery, AARE 2008 Conference Papers Collection.

Kress, G. (2010). Multimodality: A social semiotic approach to contemporary communication. New York: Routledge

Lambert, V., Coad, J., Hicks, P. y Glacken, M. (2014). Social spaces for young children in hospital. Child: care, health and development, 40(2), 195-204. DOI: 10.1111/ cch.12016

Nicholas, D. B., Fellner, K. D., Koller, D., Fontana Chow, K. y Brister, L. (2011). Evaluation of videophone communication for families of hospitalized children. Social work in health care, 50(3), 215-229. http://dx.doi.org/10.1080/00981389.2010.531998

Nisselle, A., Hanns, S., Green, J. y Jones, T. (2011). Linking and learning during hospitalisation: learning technologies connecting adolescents to school and social networks. En World Conference on Educational Multimedia, Hypermedia and Telecommunications.

Nisselle, A., Hanns, S., Green, J. y Jones, T. (2012). Accessing flexible learning opportunities: children's and young people's use of laptops in a paediatric hospital. Technology, Pedagogy and Education, 21(1), 3-20. DOI: 10.1080/1475939X.2012.659883.

Peirats Chacón, J. y Granados Saiz, J. (2015). Las unidades pedagógicas hospitalarias y el aprendizaje por proyectos de trabajo. Aula de Encuentro, 17(1), 187-211.

Peirats Chacón, J., Gallardo Fernández, I.M., San Martín Alonso, A. y Cortés i Mollà, S. (2015). Los contenidos curriculares digitalizados: Voces y silencios en el ámbito editorial. Educatio Siglo XXI, 33(3), 39-62. DOI: http://dx.doi.org/10.6018/j/240801

Pivec, M. (2007). Editorial: play and learn: Potentials of game-based learning. British Journal of Educational Technology, 38(3), 387-393.

Prendes, M. P. y Serrano, J.L. (2014). Investigando las posibilidades de las TIC en las aulas hospitalarias. En el Congreso Internacional EDUTEC: El hoy y el mañana junto a las TIC, Córdoba.

Rodríguez, M., Gromaz, M. y Hoppe, U. (2008). Conocimientos de e-learning específico para hospitales. En H. Bienzle, (Ed.), eHospital: e-learning para pacientes hospitalizados (pp. 115-125). Die Berater: Wien.

Rodríguez Rodríguez, J. y Rodríguez Regueira, N. (2016). Revisión de la investigación publicada sobre el libro de texto digital en revistas, publicaciones y congresos inter- 
Avances de la investigación en educación y TIC en aulas hospitalarias José Peirats Chacón, Jesús Granados Saiz y Diana Morote Blanco

nacionales de referencia. Profesorado, 20(1). 9-31. Recuperado de http://www.redalyc.org/articulo.oa?id=56745576001

Serradas Fonseca, M. (2011). Aprendo en el hospital: recurso instruccional multimedia para niños hospitalizados. Educere, 15(52), 683-690.

Serrano, J. L. y Prendes, M. (2011). Mejora educativa en las aulas hospitalarias. Proyecto ALTER. Trabajo presentado en Congreso Internacional EDUTEC 2011, Pachuca.

Serrano, J. L. y Prendes, M.P. (2014). TIC para la mejora educativa en aulas hospitalarias. Pixel-Bit, Revista de Medios y Educación, (45), 23-36. DOI: http://dx.doi. org/10.12795/pixelbit.2014.i45.02

Silva, G. (2012). Las vivencias de los niños hospitalizados desde sus dibujos y testimonios. Lima: Fundación Telefónica.

Leger, P. y Campbell, L. (2008). Evaluation of a school-linked program for children with cancer. Health Education, 108(2), 117-129. http://dx.doi.org/10.1108/09654280810855577

Vélez, O. y Galeano, M. E. (2002). Investigación cualitativa: estado del arte. Medellín: Universidad de Antioquia.

Vetere, F., Green, J., Nisselle, A., Dang, X. T., Zazryn, T. y Deng, P. P. (2012). Inclusion during school absence: Using ambient technology to create a classroom presence for hospitalised children. Telecommunications Journal of Australia, 62(5). doi:10.1016/j. ijhcs.2014.04.003

Vicini, S., Bellini, S., Rosi, A. y Sanna, A. (2012, June). An internet of things enabled interactive totem for children in a living lab setting. In Engineering, Technology and Innovation (ICE), 2012 18th International ICE Conference on (pp. 1-10). IEEE. DOI: 10.1109/ICE.2012.6297713

Wadley, G., Vetere, F., Hopkins, L., Green, J. y Kulik, L. (2014). Exploring ambient technology for connecting hospitalised children with school and home. International Journal of Human-Computer Studies, 72(8), 640-653. DOI: 10.1016/j.ijhcs.2014.04.003

Wilkie, K. y Jones, A. (2008). Link and learn: Students connecting to their schools and studies using ICT despite chronic illness. In Australia Association for Research in Education conference, Canberra.

Wilkie, K. y Jones, A. (2010). School ties: Keeping students with chronic illness connected to their school learning communities. In Proceedings of the New Developments in ICT and Education Conference. Amiens: International Federation for Information Processing. 